\title{
The Analysis of Attachment Styles through Interpersonal Circumplex Description
}

\author{
Wen Xue, Shouying Zhao \\ School of Educational, Guizhou Normal University, Guiyang, China. \\ Email: whitesherry@163.com \\ Received May 18 ${ }^{\text {th }}$, 2011; revised July 23 $3^{\text {rd }}, 2011$; accepted September $9^{\text {th }}, 2011$.
}

\begin{abstract}
The study explored how the dyads with different attachment styles behave towards Leary's circumplex in cooperative and competitive tasks. 100 strangers as a sample were surveyed through two experiments on computer, by using RBQ (Riverside Behavioral Q-sort) to examine their interpersonal traits through rated by experimenters. Relation Questionnaire (RQ) and ECR were also used to measure their attachment styles. There are three conclusions based on the results of this study that: 1) Individuals of different attachment styles in interpersonal interaction had shown different trend of interpersonal styles; 2) Individuals of different attachment styles take up different area in interpersonal circumplex, which proves that it is continuous not discrete of interpersonal attachment style; 3) the dyads in different sex of different attachment types showed different satisfaction in these two tasks.
\end{abstract}

Keywords: Interpersonal Circumplex, Attachment Style, Interpersonal Relationship, Dyads

\section{Introduction}

It was mentioned in Bowlby and Ainsworth's attachment theory that patterns of childhood attachment have examined in the context of adult's experience of emotion, especially in their relationships. This extension is consistent with Bowlby's assertion that the attachment mechanism is an impact "from the cradle to the grave". Actually, the continuity of infant's attachment style has further effect on individual's manifestation towards their relationships or association (such as marriage, romantic relation, the companionship). Therefore, most attachment researches have been done by the domestic and international researchers, combined with various composition of character about cognition, personality or trait. So further exploring will be continued for the correlation of this individually emotional link and individual mental state shape, interpersonal behavior, and social cognition and support, happiness, etc.

With the gradually in-depth social science researches on adult interpersonal attachment, studies for attachment methods and means of measurement have become more diversified. In China, the main measurement of interpersonal attachment is in two ways. In the first way, individual's attachment style was measured to determine the differences between individuals with different attachment styles; and the Ladder is the determination of the one's score on attachment dimensions. Individuals' scores on different dimension will be used to determine the extent of their attachment avoidance and anxiety and so on. Questionnaires are highly applied in researches concerning both the degree in dimension structure and attachment styles. Which is used the most is the type of Adult Attachment Styles Questionnaire.

Early questionnaire survey towards adult attachment was focused on the discrete types (Hazan \& Shaver, 1987), which was developed by Ainsworth's three infant attachment types. Since then, a large number of adult attachment scales were emerging, greatly promoting the research progress in this field. According to Bowlby's theory, the individual was referred to the selfmodel and other-model, because individual's attachment style depends on their own positive or negative assessment about themselves and their supporters (especially parents). So the adult attachment styles based on the integration of these two models, can be divided in four-categorical scheme, such as secure, ambivalent, fearful and dismissing. Therefore, the subsequent establishment of attachment scale towards two dimensions-self-model and other-model, became the core content discussed by the researchers. The individual with secure attachment of cherish both the positive self-evaluation and other-evaluation, and they want others to response their own emotional needs; compared with that, ambivalent attachment style owes negative self-model, whereas of positive other-model; The individuals of dismissing attachment style possess positive self-model, but they believe that others are not worth trusting; The fearful attachment's self-model and the other-model were both negative. Based on this theoretical analysis, Bartholomew and Horowitz developed a self-report scale-Relationship Questionnaire.

Since then, based on the RQ, Brennan developed a standard adult intimate relationships Attachment Scale-Closed Relation Experience scale (ECR), a scale of 36 questions, in which, attachment anxiety and attachment avoidance as its the two dimensions composition. Currently, Attachment investigation would be confirmed by of the combination of these two item polls or they may use the ECR to determine the subjects' style while the RQ as the criterion for test.

\section{Question}

However, recent research suggests that rather than a three-or four-categorical, a dimensional, more comprehensive model may better represent adult attachment (Crowell, Fraley, \& Shaver 1999). Hazan and Shaver (1987) found that "Secure attachment tend to describe their parents friendly and warm, whereas Anxiety-Ambivalent attachment of the individuals described their families aggressive, and avoidant person tend to report their parents cold and rejecting”. Therefore, it is no doubt the measurement method based on interpersonal relationship 
can be applied to the adult attachment study. For example, Shaver and Brennan (1992) examined the association of attachment with the Big Five personality model (FFM). According to their study, "more securely attached participants reported higher extroversion and agreeableness. Contrarily, those individuals with anxious attachment style more likely to report lower agreeableness, and higher neuroticism”.

Therefore, preliminary research, at least, proofed that adult attachment style, to some extent, is related to the individuals' interpersonal traits or personality, interpersonal communication and social interaction. That is why insecure attachment person may show a hostile or compliance attitude in their interpersonal communication with others. The Figure 1 indicates the relations between four attachment styles and two dimensions-interpersonal anxiety and avoidance. It can also be observed from this figure that the continuous space between these four attachment styles is not mutually discrete separate, but some among them have not been explored. The independence of these four styles gives us some illusion that they are separate. Indeed, participates are distributed around all the space, even the area between two lines of canonical four styles. That is the reason why some individuals with Preoccupation attachment style would be changed into Secure after they fell in love or got marriage, since they may locate originally between these two types.

In social interaction, a successful communication is always attributed to the interpersonal behavior of reciprocity or complementarity of two parnters. Established by this theory, Interpersonal Circumplex can accurately predict the successful maintaining rate of a relationship between two peoples. The IPC is divided by two dimensions, control and affiliation, into eight parts. They are assured-dominant (PA), in the anticlockwise order, arrogant-calculating (BC), cold-hearted (DE), aloofintroverted (FG), unassured-submissive (HI), unassuming-ingenuous (JK), and warm-agreeable (LM), and (NO) octants (see Figure 2). IPC attempts to describe and explain the character of a person, especially his or her interpersonal style. Carson (1969) suggested that only the Agreeableness dimension is similar to the position in the leading position of the interpersonal dimension of the two sides complement each other to attract each other.

The two dimensions of The FFM that-extraversion and agreeableness-are very similar to the affiliation and domnance

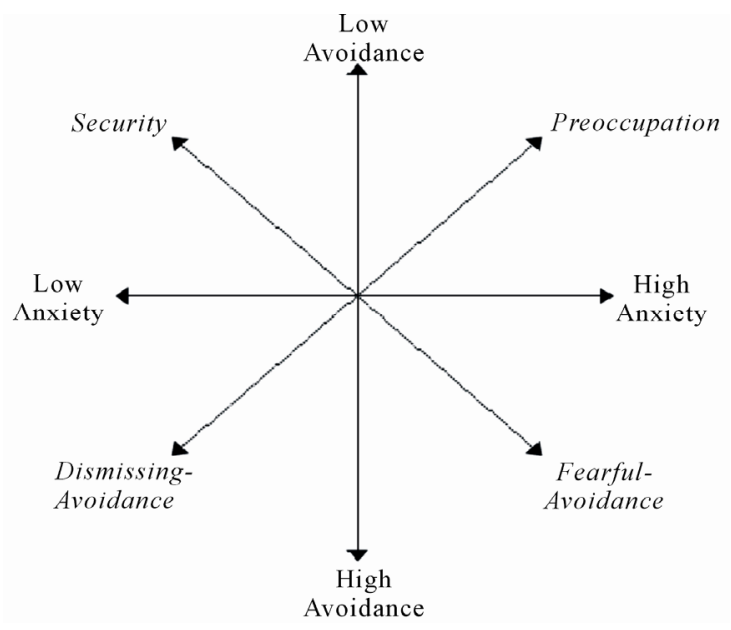

Figure 1.

The relation between attachment style and two dimensions-interpersonal avoidance and anxiety.

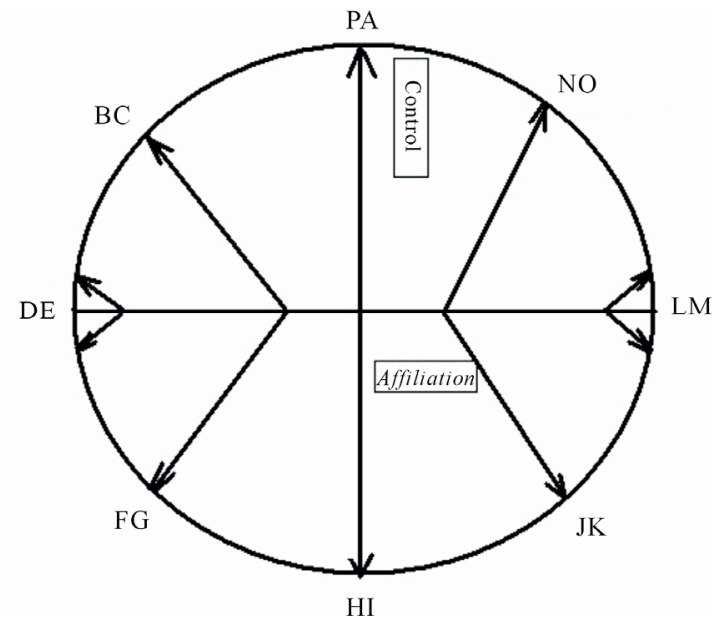

Figure 2.

Leary's interpersonal circumplex.

of IPC, respectively (McCrae \& Costa, 1989). Therefore, utilizing the previous theory, we intend to adopt experimental tasks with the questionnaire method, in order to examine the description of Leary's interpersonal Circumplex model for interpersonal attachment of dyads with opposite sex in different tasks. And explore the association of individual's interpersonal attachment and interpersonal traits, while analysis the satisfaction of dyads with different attachment styles matching in different tasks.

\section{Method}

\section{Subjects}

Subjects were 50 men and 50 woman students at several schools in Guizhou, some of whom came from high school, and others are graduates or undergraduates. They were ranged in age from $16 \sim 30$. They were matched in pairs in random, and each pair contains a man and a woman. There are two experimental situations, the cooperative situation and competitive situation, in each of which 25 dyads participated.

\section{Performance task}

In cooperative situation, each dyad was surveyed through an experiment of detective game on computer, in which 13 things were asked to be found by their cooperation. And in competetive situation, each dyad was surveyed through an experiment of combat game on computer, in which they should use their skills or ability to defeat their partner.

\section{Measure}

After telling the instruction about these two tasks, the experimenters ran the detective or combat FLASH game on computer, and asked the opposite-sex dyad to complete it together. Each interaction lasted approximately $3 \mathrm{~min}$, while the experimenters used RBQ (Riverside Behavioral Q-sort) to examine their interpersonal traits.

\section{Results and Analysis}

\section{The Distribution of Individuals with Different Attachment Types in IPC}

By the end of the experiment, subjects should complete the 
self-report scale RQ and ECR also, in order to determine their attachment styles. There are 43 people of secure attachment, 15 of fearful attachment, whereas 22 subjects of ambivalent attachment, and 20 with dismissing style. Then the subjects' attachment type was tested with their highest scores in the eight subscales of RBQ through correlation analysis. Every subscale would represent a trait in the circumplex, the pie charts are shown as follow (see Figure 3).

Through the Figure 3, subjects with secure attachment tested in two tasks mainly reflects a highly-frequency performance in PA, JK LM, and NO. Among other three insecure attachment styles, it is easy to find that the interpersonal performance of ambivalent-attachment individual is similar to the secure one, but the proportion of each trait is distinguished with it. Fearful attachment were tested mainly in interpersonal style of BC, DE, and FG, which is the most distinguished on compared with the secure style ; while the subjects of dismissing attachment got high scores in FG and JK.

In order to exclude the possibility that extreme data would be generated by the highest scores, and to further explore the relationship between variables about the 4 types of attachment and the 8 interpersonal traits in IPC octant, we tested the data of 100 individual of these two tasks through Pearson two-tailed test (Table 1 ).

It can be well informed in Table 1 that subjects with secure attachment style (A) was more obvious in the performance of BC, PA, NO, LM in interpersonal interaction, which shows significantly positive correlation in PA, but shows a signifycantly negative correlation with the HI. This matches the hypothesis that highly positive self-model is the main character of secure individuals. When it comes to fearful attachment style (B), the situation may become just opposite, which shows positive relation towards the traits of BC, DE, FG, HI and a lower scores on the affiliation dimension of the circumplex, while a higher score in complaisance, because individuals under this style take a part in negative self-model and other-model. Subjects with ambivalent attachment take a positive correlation with BC and DE two traits. Although it may indicate a conflicttion against the highest-score pie chart that DE takes no evi-

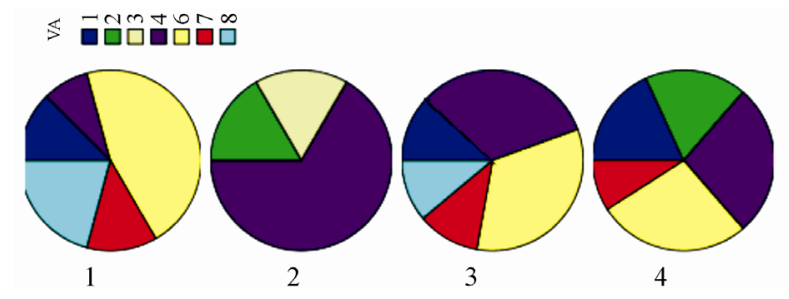

Figure 3.

The highest score's distribution of RBQ in different attachment style individuals. (Pies: 1-secure; 2-fearful; 3-ambivalent; 4-dismissingTrait: from 1-PA to 8-NO).

Table 1.

The correlation coefficent of eight interpersonal traits and attachment styles in two tasks.

\begin{tabular}{ccccccccc}
\hline & PA & BC & DE & FG & HI & JK & LM & NO \\
\hline A & $0.27^{* *}$ & $0.23^{*}$ & -0.07 & -0.18 & $-0.27^{* *}$ & 0.09 & 0.14 & 0.17 \\
B & -0.13 & -0.13 & 0.013 & $0.31^{* *}$ & 0.03 & 0.01 & -0.06 & -0.09 \\
C & -0.13 & -0.04 & 0.089 & 0.03 & -0.01 & 0.03 & 0.07 & -0.10 \\
D & 0.01 & 0.025 & $-0.22^{*}$ & -0.04 & -0.01 & 0.09 & 0.02 & 0.01 \\
\hline
\end{tabular}

dence above this attachment style, it can be explained that this characteristics is due to this type of attachment and subjects' average score on this trait are not low, but this is not their highest score characteristics. What should be a surprise is that the dismissing attachment shows similarity with the secure on. Since individuals with this type although evaluate others negative or not worth trusting, a positive self-model was formalized in their inner mechanisms of attachment experience in early stage. So they may also get a not low score on control dimension, however, they may cherish less ability of an agreeable nature, which can be indicated on the decreased correlation coefficient compared with the secure attachment, because their other-model are negative.

To a contrary, Figure 4 provides a clear distribution of different attachment styles' location in the circumplex. And each style is depicted as a consecutive manner in the circumplex, which proofs the continuum of attachment style shown in horizontal and vertical dimensions. Among them, three types of attachment are in continuous interpersonal performance, without being interrupted, whereas ambivalent attachment person take a break in the circular model. In this interpersonal process, individuals belonged to ambivalent attachment style, tended to be divided into two forms, one of which relatively came closer to fearful attachment, and the other form performed relatively as the secure one, which also well confirmed the assumption that it is the continuity rather than separation should be the nature of attachment styles. But whether it is close to the fearful attachment, or secure attachment, it showed high compliance more than the dominance on the control dimension. In our daily life it often seems as manifestation as lacking of confidence when put it in social relation. This should be attributed to the negative self-evaluation model of ambivalent attachment.

\section{The Satisfaction of Dyads with Different Attachment Styles in Cooperation Task}

Calculating through variance analysis, the statistic signifycance $($ Sig) $=0.018<0.05$ (Table 2), indicated that, between dyads with the different attachment-style matching, at least one group showed significant difference towards other groups. As shown in the satisfaction mean picture (Figure 5), both partners in the dyads were secure attachment showed the most cooperation satisfaction in this interaction, followed which is the dyads

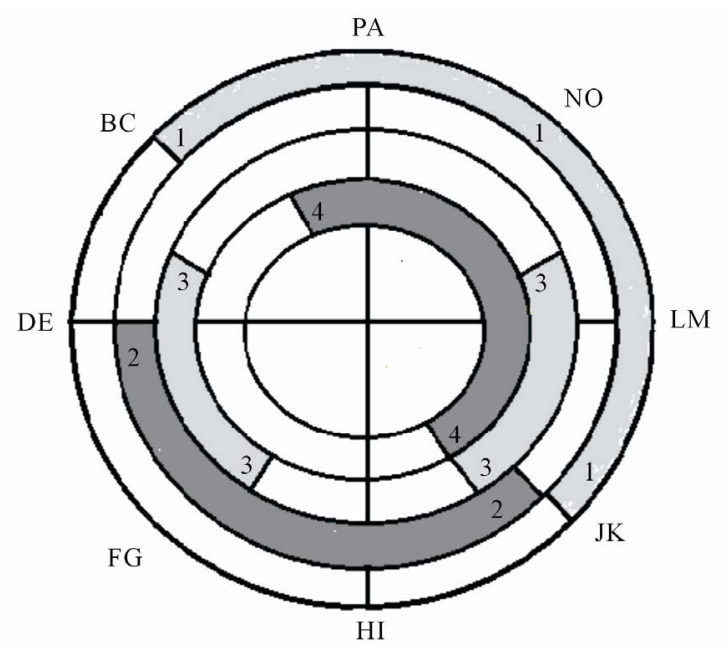

Figure 4.

The description of four attachment styles in IPC (1-secure; 2-fearful; 3-ambivalent; 4-dismissing). 
with only one secure attachment individual, and dyads with both are insecure attachment took the lowest score compared with the other two group.

\section{The Satisfaction of Dyads with Different Attachment Styles in Competition Task}

Similar to the cooperative task, the statistic significance (Sig) in competitive task is $0.018<0.05$ (see Table 3), which also indicated at least one group showed significant difference towards other groups. We can be well informed through the linear figure (Figure 6), that dyads of secure attachment showed significantly higher satisfaction than dyads with insecure satisfaction. But compared to cooperative tasks, the scores between the two groups with insecure attachment style, the difference is not distinguished. In cooperation task, to some kind of degree, increase peoples' satisfaction when it comes to exact social association.

\section{Conclusion}

Three conclusions based on the results of this experimental

Table 2.

Variance analysis for cooperative satisfaction degree towards dyads in different attachment styles.

\begin{tabular}{cccccc}
\hline \multicolumn{5}{c}{ ANOVA } & \\
\hline & Sum of squares & Df & Mean of squares & F & Sig. \\
Between groups & 10.183 & 2 & 473.895 & 3.256 & 0.018 \\
In group & 23.257 & 22 & 145.524 & & \\
Sum & 33.440 & 24 & & & \\
\hline
\end{tabular}

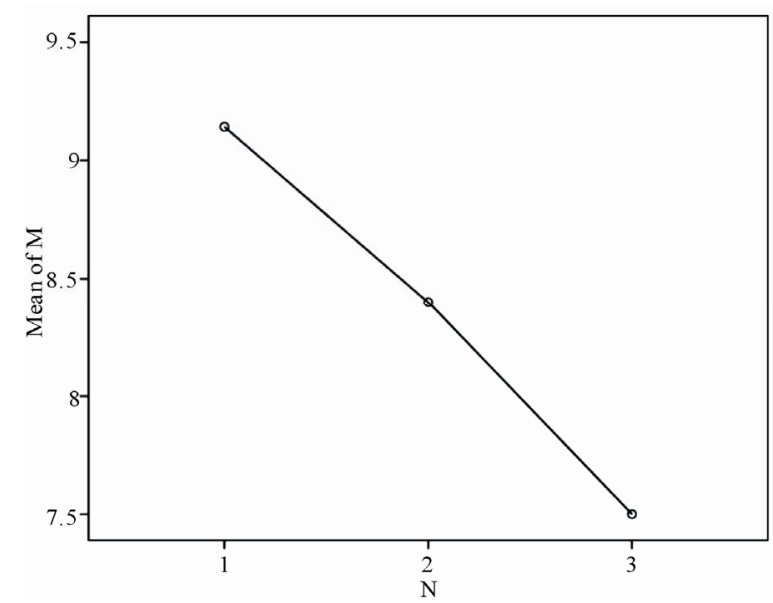

Figure 5.

Average score of cooperative satisfaction degree towards dyads in different attachment styles.

Table 3.

Variance analysis for competitive satisfaction degree towards dyads in different attachment styles.

\begin{tabular}{ccccccc}
\hline \multicolumn{6}{c}{ ANOVA } & \\
\hline & Sum of squares & Df & Mean of squares & F & Sig. \\
Between groups & 11.248 & 2 & 473.895 & 4.667 & 0.020 \\
In group & 26.512 & 22 & 145.524 & & \\
Sum & 37.760 & 24 & & & \\
\hline
\end{tabular}

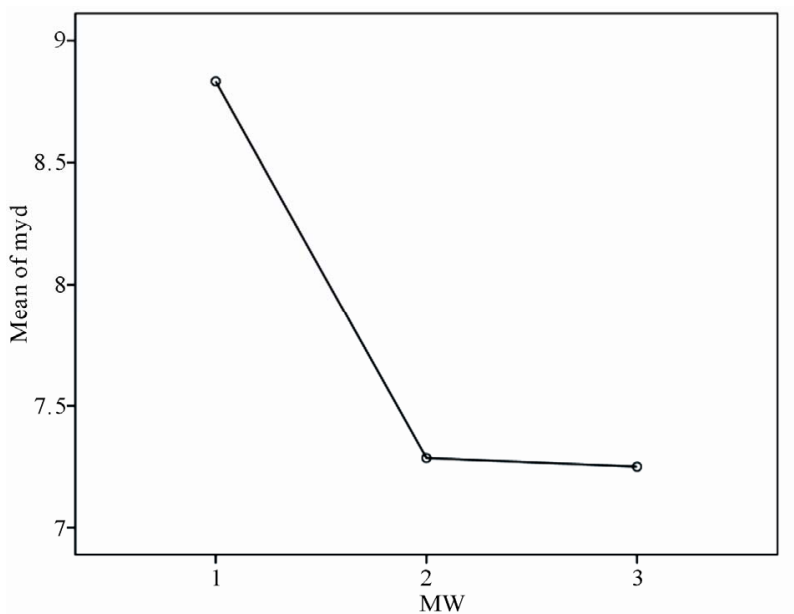

Figure 6.

The average score of competitive satisfaction degree towards dyads in different attachment styles. (1-dyads of secure; 2-dyads with only one insecure partner; 3-dyads of unsecure).

study can be showed that: 1) Individuals of different attachment styles in interpersonal interaction had shown different trend of interpersonal styles; 2) Individuals of different attachment styles take up different area in interpersonal circumplex, which proves that it is continuous not discrete of interpersonal attachment style; 3 ) the dyads in different sex of different attachment types showed different satisfaction in these two tasks. In cooperative task, dyads of secure attachment style took the highest satisfaction, and of insecure attachment style with the lowest one, while in competitive task, it is not distinguished between dyads of insecure attachment style and dyads with only one insecure partner.

\section{References}

Gallo, L. C. et al. (2003). An interpersonal analysis of adult attachment style: Circumplex descriptions, recalled developmental experiences, self-representations and interpersonal functioning in adulthood. Journal of Personality, 71, 141-182.

doi:10.1111/1467-6494.7102003

Li, T. G. (2005). Attachment and memory for emotional events. Journal of Peking University, 41, 965-974.

Ansell, E. B., Kurtz, J. E., \& Markey, P. M. (2008). Gender differences in interpersonal complementarity within roommate dyads. Personality and Social Psychology Bulletin, 34, 502-512.

doi:10.1177/0146167207312312

Gurtman, M. B., \& Pincus, A. L. (2000). Interpersonal adjective scales: Confirmation of circumplex structure from multiple perspectives. Personality and Social Psychology Bulletin, 26, 374-384. doi:10.1177/0146167200265009

Markey, P. M., Funder, D. C., \& Ozer, D. J. (2003). Complementarity of interpersonal behaviors in dyadic interactions. Journal of Personality and Social Psychology, 29, 1082-1090.

Mayseless, O., \& Scharf, M. (2007). Adolescents' attachment representations and their capacity for intimacy in close relationships. Journal of research on adolescence, 17, 23-50.

Sadler, P., \& Woody, E. (2003). Is who you are who you're talking to? Interpersonal style and complementarity in mixed-sex interactions. Journal of Personality and Social Psychology, 84, 80-96. doi:10.1016/j.jecp.2004.02.002

Sullivan, H. S. (1953). The interpersonal theory of psychiatry. New York: Norton.

Zimmermann, P. (2004). Attachment representations and characteristics of friendship relations during adolescence. Journal of Experimental Child Psychology, 88, 83-101. doi:10.1016/j.jecp.2004.02.002 